University of Texas Rio Grande Valley

ScholarWorks @ UTRGV

$1-2017$

\title{
Fish Oil with Higher DHA Content and Voluntary Exercise Decreases Postmenopausal Bone Loss
}

Jameela Banu

The University of Texas Rio Grande Valley

Gabriel Fernandes

Follow this and additional works at: https://scholarworks.utrgv.edu/bio_fac

Part of the Alternative and Complementary Medicine Commons, and the Biology Commons

\section{Recommended Citation}

Banu J, Fernandes G (2017) Fish Oil with Higher DHA Content and Voluntary Exercise Decreases Postmenopausal Bone Loss. J Osteopor Phys Act 5: 188. doi: 10.4172/2329-9509.1000188

This Article is brought to you for free and open access by the College of Sciences at ScholarWorks @ UTRGV. It has been accepted for inclusion in Biology Faculty Publications and Presentations by an authorized administrator of ScholarWorks@ UTRGV. For more information, please contact justin.white@utrgv.edu,william.flores01@utrgv.edu. 


\title{
Fish Oil with Higher DHA Content and Voluntary Exercise Decreases Postmenopausal Bone Loss
}

\section{Jameela Banu ${ }^{1 *}$ and Gabriel Fernandes ${ }^{2}$}

${ }^{1}$ Department of Health and Biomedical Sciences and Department of Biology, University of Texas Rio Grande Valley, Edinburg, Texas, USA ${ }^{2}$ Department of Medicine, University of Texas Health Science Center, San Antonio, Texas, USA

\begin{abstract}
There is increasing evidence suggesting that fish oil (FO) decreases bone resorption by reducing osteoclastogenesis and regular exercise (EX) increases bone mass. EX is associated with increasing bone formation. Therefore, the combined effects of FO intake and EX may have additive effects by both increasing bone formation and decreasing bone resorption. To demonstrate this, we studied the effects of FO and EX on the bone of mice after an ovariectomyinduced bone loss.

Twelve months old C57BL/6 female mice were either sham operated or ovariectomized, divided into different dietary and EX group and maintained for 3 months before sacrifice.

The distal femoral metaphysis (DFM) showed significantly higher total BMC in both the FO sham sedentary groups. $30 / 20$ ovariectomized sedentary mice had higher total bone mineral content (BMC). EX significantly increased BMC in the control and one of the FO ovariectomized (18/12) groups. The total bone mineral density (BMD) was higher in both the FO ovariectomized mice. EX significantly increased BMD in the control and combination with 18/12 ovariectomized groups. Cortical BMC increased after EX with both FO diets.

The DFM showed no significant changes in the trabecular thickness. However, the trabecular number increased in mice fed both FO diet and on EX. Trabecular separation was decreased in the control and 30/20 FO-fed animals but increased in the 18/12 FO-fed mice.

In conclusion, in the distal femoral metaphysis, FO-fed mice showed increased BMD. In combination with EX, the 30/20 FO-fed mice showed higher trabecular number and cortical bone mass as well as decreased trabecular separation.
\end{abstract}

Keywords: Fish oil; Postmenopausal bone loss, pQCT; $\mu \mathrm{CT}$ densitometry

\section{Introduction}

Osteoporosis is a disease primarily associated with aging in men and menopause in women. The hallmark of this medical condition is that minor trauma causes fractures as the bones are very fragile. Bones are dynamic organs that undergo modeling and remodeling as vertebrate organisms grow, mature and age, via specialized cells called osteoblasts and osteoclasts [1]. Bone remodeling maintains the skeleton by adding to growing bone, repairing damaged portions and removing old bone. Bones also are the body's reservoir depots for calcium and phosphorus, and both can be released into the blood to maintain ionic homeostasis. Bone loss occurs when there is an imbalance in the process of bone remodeling. This imbalance is because there is increased bone resorption alone or this is combined with decreased bone formation.

Therefore, treating and/or preventing bone loss will be more effective if it is focused on the reduction of bone resorption (osteoclast activity) and in increasing bone formation (by reactivation or maintenance of osteoblasts). Currently, several therapeutics are available to treat and prevent osteoporosis. However, the side effects of these drugs can outweigh the benefits in some patients [2-5] which has increased interest in finding alternative medicines to treat and prevent osteoporosis.

Essential fatty acids like omega 3 fatty acids have been shown to protect bones from postmenopausal bone loss [6-9]. Salt water fish is a major source of omega-3 fatty acid. Fish oil (FO) inhibits osteoclastogenesis, thereby, decreasing bone resorption. Two of the long chain omega-3 fatty acids are eicosapentaenoicacid (EPA) and docosahexaenoicacid (DHA) have shown different effects on bone [10] with DHA showing more bone protective properties than EPA. In this study, we tested two different combinations of EPA/DHA to determine their effects on bone after ovariectomy.

As age advances, there is a reduction in physical activity and this may also contribute to increased bone loss. It is very well established that increase in physical activity or exercise has beneficial effects on bone mainly by stimulation of bone formation [11]. However, moderate voluntary exercise has the most benefits on bone. A common form of voluntary exercise used on rodent animal models is treadmill running, as this can produce high bone strains of normal coordinated activity [12-14]. In the present study, we used both FO (two different EPA/ DHA ratios) and flatbed treadmill exercise to determine the effects on the distal femur of middle-aged ovariectomized mice.

*Corresponding author: Jameela B, Department of Health and Biomedica Sciences and Department of Biology, College of Health Affairs and College of Sciences, University of Texas Rio Grande Valley, Edinburg, TX, USA, Tel: 9566653222; Fax: 9566655253; E-mail: jameela.banu@utrgv.edu

Received December 23, 2016; Accepted January 18, 2017; Published January 28, 2017

Citation: Banu J, Fernandes G (2017) Fish Oil with Higher DHA Content and Voluntary Exercise Decreases Postmenopausal Bone Loss. J Osteopor Phys Act 5 188. doi: $10.4172 / 2329-9509.1000188$

Copyright: @ 2017 Banu J, et al. This is an open-access article distributed unde the terms of the Creative Commons Attribution License, which permits unrestricted use, distribution, and reproduction in any medium, provided the original author and source are credited. 


\section{Materials and Methods}

\section{Animals}

Twelve months old C57BL/6 female mice, were bought from Jackson Laboratory (Bar Harbor, ME). After two weeks of acclimatization, they were either sham-operated or ovariectomized and divided into the following groups: Group 1 Lab chow sham sedentary (LC S Sed); Group 2 LC S exercise (LC S EX); Group 3 LC ovariectomized S (LS O Sed); Group 4 LC O EX; Group 5 18/12 S Sed; Group 6 18/12 S EX; Group 7 18/12 O Sed; Group 8 18/12 O EX; Group 9 30/20 S Sed; Group 10 30/20 S EX; Group 11 30/20 O Sed; Group 12 30/20 O EX. Control group was fed and maintained on Lab Chow diet (AIN 93) and the treatment groups were fed experimental diets containing two different types of 5\% fish oil (18/12 and 30/20), containing either EPA $18 \%$ and DHA $12 \%$ or $30 \%$ EPA and 20\% DHA, added to the lab chow and mixed well.

Mice were on their respective diets and a treadmill exercise regimen three days after surgery. The mice were on their treatments for 3 months and then sacrificed. The femur was removed and stored for PQCT and $\mu \mathrm{CT}$ densitometry. The experiments were approved by UTHSCSA's IACUC committee. All the procedures conducted were in accordance with the ethical standards of $\mathrm{NIH}$ and the University at which the studies were conducted.

\section{Body weight and organ weights}

At the beginning of the experiment, mice were weight matched using a CS 200 (Ohaus, Pine Brook NJ) balance. They were also weighed at the time of sacrifice. At the time of euthanasia, several organs including the uterus, adipose tissue, quadriceps and gastrocnemius, liver, spleen and kidneys were weighed using a Mettler Balance (Columbus, $\mathrm{OH}$ ), at the time of sacrifice.

\section{Peripheral quantitative computerized tomography densitometry (pQCT)}

Cortical and cancellous bones of the distal femoral metaphysis (DFM) were analyzed using pQCT densitometry (XCT Research M system, Norland Stratec, Birkenfeld, Germany), as described previously $[11,15]$. Five slices were scanned including the growth plate. Three slices $1 \mathrm{~mm}$ proximal to the knee joint were analyzed. The following parameters were determined: cancellous bone mineral content ( $\mathrm{Cn}$ $\mathrm{BMC})$, cancellous bone mineral density (Cn BMD), cortical bone area (Ct Ar), cortical BMC (Ct BMC), cortical BMD (Ct BMD), cortical thickness (Ct Th), periosteal perimeter (Peri PM) and endocortical perimeter (Endo PM).

\section{Micro computed tomography $(\mu \mathrm{CT})$}

The distal femoral metaphysis was scanned ( $\mathrm{n}=6$ from each group) using a high resolution $\mu \mathrm{CT}$ scanner Xradia $\mu$ XT200 (Xradia Inc, Concord, CA) at $20 \mu$. All images were acquired using standard parameters: resolution of $9.1 \mu \mathrm{m}, \mathrm{x}$-ray source of $90 \mathrm{KV}$, power of $4.0 \mathrm{~W}$ and current of $44.0 \mu \mathrm{A}$ with an exposure time of $12 \mathrm{~s}$ per slice. The scans were analyzed using Tri/3D Bon (Ratoc, Inc., Japan) for the following parameters: Total Volume (TV), Bone Volume (BV), BV/TV, Trabecular Number ( $\mathrm{Tb} \mathrm{N}$ ), Trabecular Thickness ( $\mathrm{Tb} \mathrm{TH})$, Trabecular Separation (Tb S), connectivity density (Conn Den).

\section{Statistical analysis}

Data were analyzed by one-way ANOVA and unpaired t-test using GraphPad Prism 4 (GraphPad Software Inc, San Diego, CA, USA). Results are expressed as Mean \pm SE. $\mathrm{P} \leq 0.05$ was considered to be significant. Newman-Keuls multiple comparison test was used to analyze the differences between groups for significance.

\section{Results}

\section{Body weight and organ weights}

Effects of fish oil: Final body weight did not change significantly although the animals in the FO groups were heavier than those in the lab chow group (Figure 1ii). Mice in the FO-fed groups had significantly increased adipose tissue when compared to that of the LC fed mice (Figure 1iii). Ovariectomized mice from all groups had significantly smaller uterus (Figure 1iv).

Effects of exercise: Exercise did not change the body weight in all the groups studied (Figure 1ii). Although exercise decreased adipose tissue in all the exercise groups, this decrease was significant only in the 18/12 S EX and 30/20 S EX groups (Figure 1iii). The weight of the uterus was further decreased in the EX groups (Figure 1iv).

\section{pQCT densitometry}

Effects of fish oil: Total BMC was significantly higher in the LC O EX and 30/20 O Sed groups when compared to that of LC O Sed groups (Figure 2i). Both the FO groups showed significantly higher total BMC in the sham Sed groups. Higher total BMD was seen in the 30/20 S Sed and 30/20 O Sed groups, but this increase was not statistically significant (Figure 2ii). Significant increases in the cortical BMC were seen in the $30 / 20$ S Sed group when compared to that of LC S Sed group (Figure 2iii). However, there was no significant change in the cortical BMD (Figure 2iv), cortical thickness, periosteal perimeter and endocortical perimeter in any of the groups studied (Figure 3i-3iii).

Effects of exercise: Significant increase in the total BMC was seen in the LC O EX, 30/20 S Sed, and the LC O EX groups (Figure 2i). The total BMD increased in mice from 18/12 O EX ( $p<0.08)$ (Figure 2ii). Mice from the LC O EX group showed significantly higher cortical BMC when compared to that of LC O Sed mice (Figure 2iii). Similarly, significantly higher cortical BMC was observed in mice from the $18 / 12$ O EX group, when compared to that of $18 / 12$ O Sed. Cortical BMD did not change significantly in any of the groups studied (Figure 2iv). Cortical thickness increased in the 30/20 S EX animals than LC S EX group (Figure 3i). Similarly, 18/12 S EX mice showed higher cortical thickness, when compared to that of $18 / 12 \mathrm{~S}$ Sed and barely increased $(\mathrm{p}<0.06)$, than LC S EX group mice (Figure $3 \mathrm{i})$.

\section{$\mu \mathrm{CT}$ densitometry}

Effects of fish oil: Higher trabecular number was seen in mice from 18/12 S EX group, while those in the 30/20 fed mice, showed increased trabecular number in the OVX groups (Figure 4i). There were no changes in the trabecular thickness was observed in any of the groups studied. Mice from 18/12 fed group showed higher trabecular separation, while those from 30/20 group showed less trabecular separation (Figure 4iii).

Effects of exercise: Significantly higher trabecular number was seen in the 18/12 S EX as compared to 18/12 S Sed and LC S EX (Figure 4ii). There was increased trabecular number in the 30/20 O EX group, but this increase was not statistically significant (Figure 4ii). No significant difference in the trabecular thickness was seen in any of the groups studied (Figure 4i). EX decreased trabecular separation in the FO treated sham and OVX groups (Figure 4iii).

\section{Discussion}

Dietary fatty acids have significant beneficial effects on bone [16] 

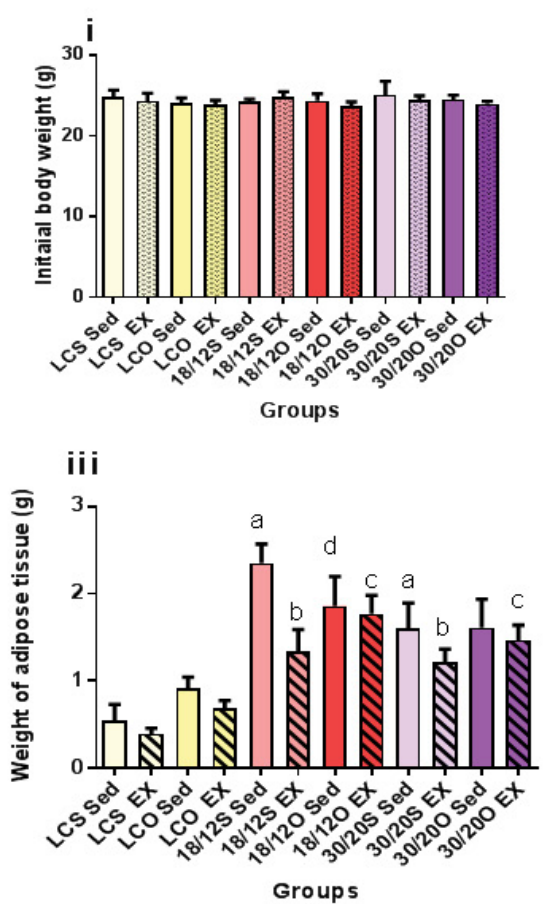

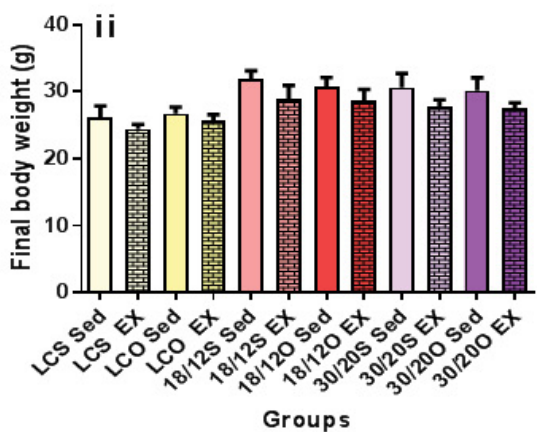

iv

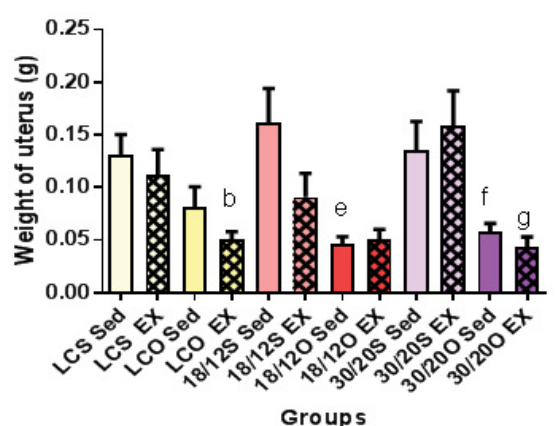

Groups

${ }^{a} p \leq 0.05$ vs LC S Sed; ${ }^{b} p \leq 0.05$ vs LC SEX, ${ }^{c} p \leq 0.05$ vs LC O EX; ${ }^{d} p \leq 0.05$ vs LC O Sed; ${ }^{e} p \leq 0.05$ vs $18 / 12$ $S$ Sed; ${ }^{f} p \leq 0.05$ vs 30/20S Sed; ${ }^{g} p \leq 0.05$ vs $30 / 20$ SEX

Figure 1: Effects of fish oil on body weight and organ weights of middle aged female C57BI/6 Mice: (i) Initial body weight; (ii) Final body weight; (iii) Weight of adipose tissue; (iv) Weight of uterus (ap $\leq 0.05$ vs. LC S Sed; bp $\leq 0.05$ vs. LC S EX, cp $\leq 0.05$ vs. LC O EX; dp $\leq 0.05$ vs. LC O Sed; ep $\leq 0.05$ vs. $18 / 12$ S Sed; fp $\leq 0.05$ vs. $30 / 20$ S Sed; gp $\leq 0.05$ vs. $30 / 20$ S EX).
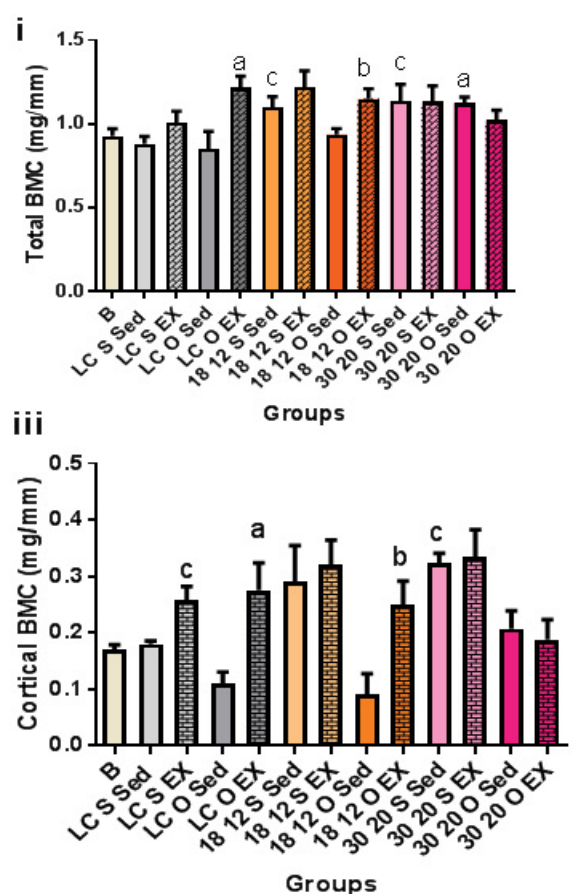

${ }^{a} \mathrm{p} \leq 0.05$ vs LC O Sed; ${ }^{b} \mathrm{p} \leq 0.05$ vs $18 / 12$ O Sed; ${ }^{c} p \leq 0.05$ vs LC S SED; ${ }^{p} p \leq 0.05$ vs LC O Sed; ${ }^{*} p=0.06$ vs LC S EX; ${ }^{*} p=0.08$ vs LC S Sed

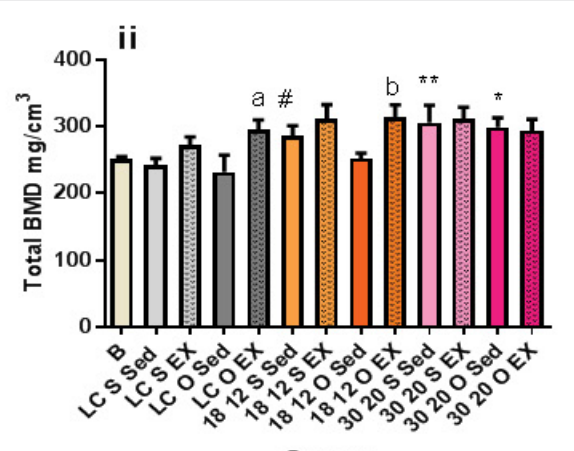

iv

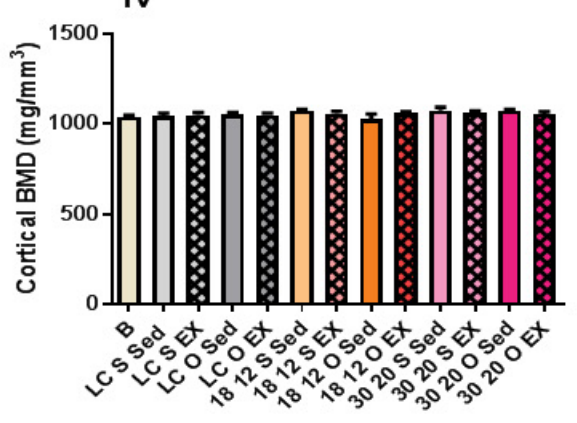

Groups

Figure 2: Effects of Fish Oil on the Total and Cortical Bone Parameters of the Distal Femoral Metaphysis of Middle Aged C57Bl/6 Mice (pQCT): (i) Total BMC; (ii) Total BMD; (iii) Cortical BMC (iv) Cortical BMD (ap $\leq 0.05$ vs. LC O Sed; bp $\leq 0.05$ vs. $18 / 12$ O Sed; cp $\leq 0.05$ vs. LC S SED; * $\leq 0.05$ vs. LC O Sed; \#p=0.06 vs. LC S EX; ${ }^{* *} p=0.08$ vs. LC Sed). 

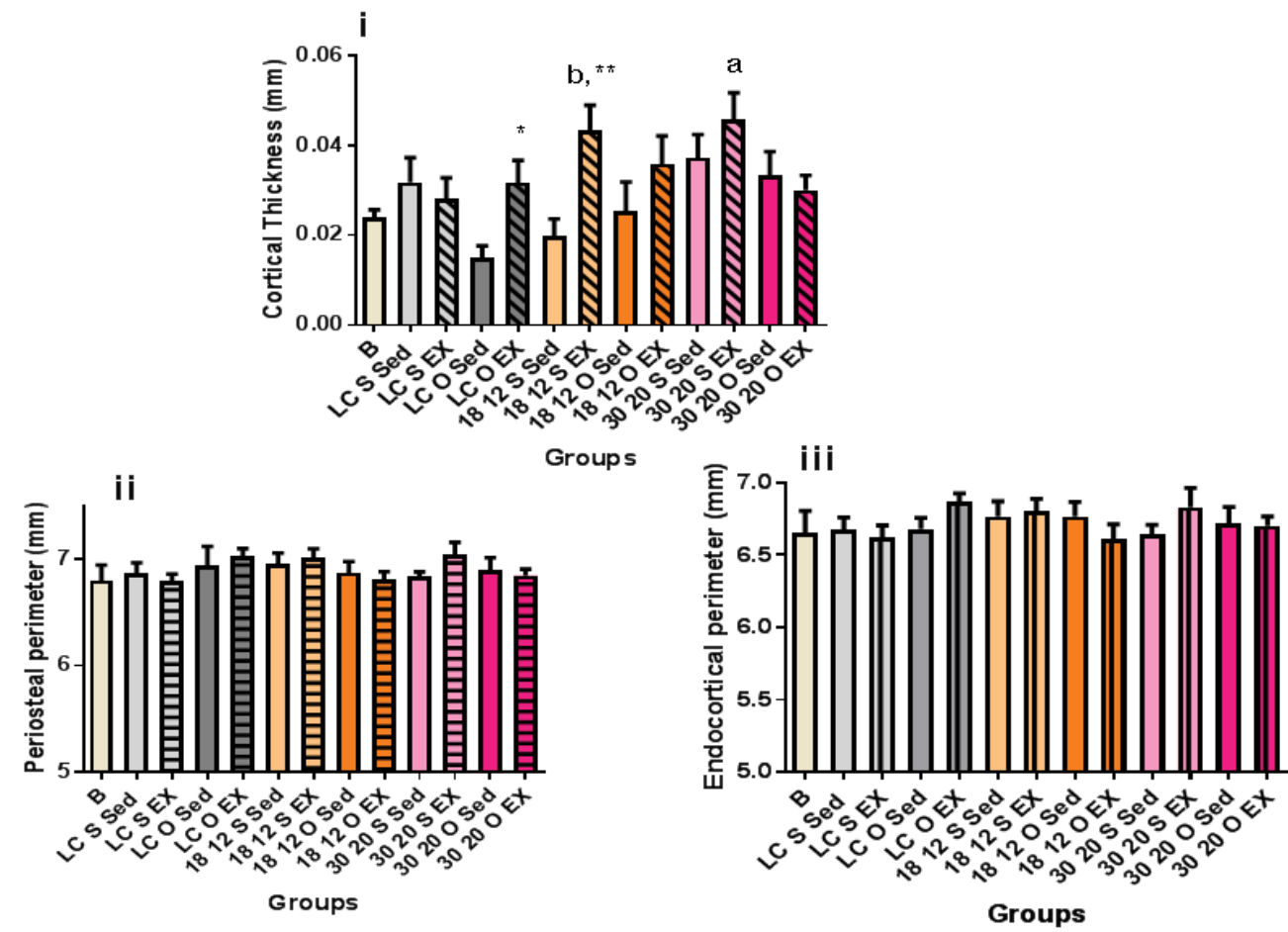

${ }^{a} \mathrm{p} \leq 0.05$ vs LC S EX; ${ }^{b} \mathrm{p} \leq 0.05$ vs $18 / 12$ S Sed; ${ }^{*} \mathrm{p}=0.06$ vs LC O Sed; ${ }^{*} \mathrm{p}=0.06$ vs LC S EX

Figure 3: Effects of fish oil on the cortical thickness and perimeters of the distal femoral metaphysis of middle aged C57BI/6 mice (pQCT): (i) cortical thickness; (ii) Periosteal perimeter; (iii) Endocortical perimeter (ap $\leq 0.05$ vs. LC S EX; bp $\leq 0.05$ vs. $18 / 12$ S Sed; ${ }^{*} p=0.06$ vs. LC O Sed; ${ }^{* *} p=0.06$ vs. LC S EX).

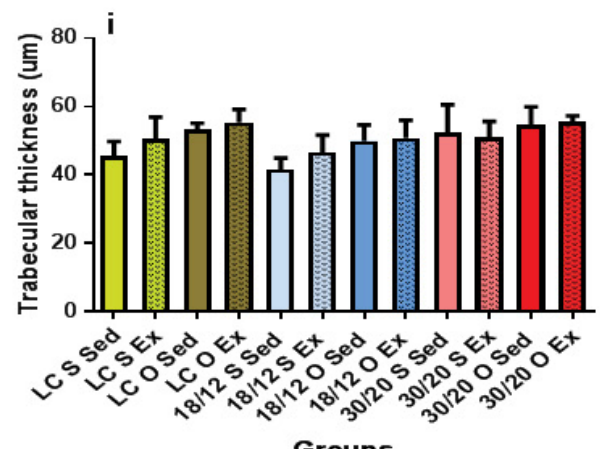

Groups
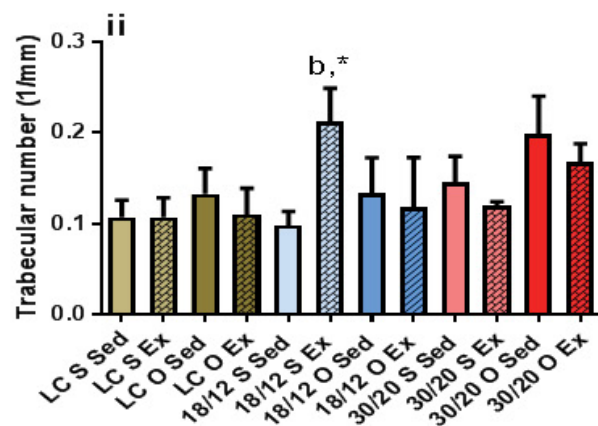

Groups

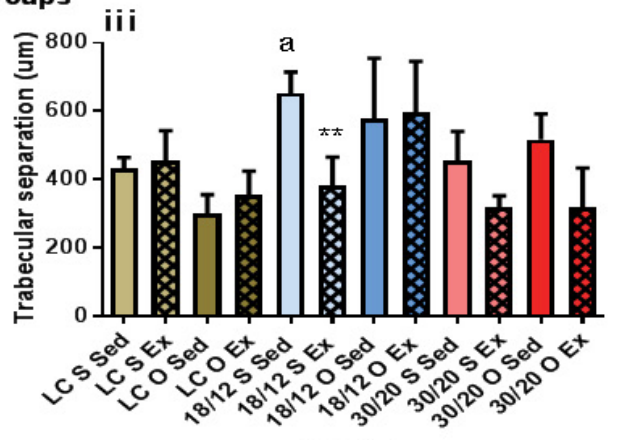

Groups

$a_{p} \leq 0.05 \vee s$ LC S Sed; ${ }^{b} p \leq 0.05 v s 18 / 12$ S Sed; ${ }^{*} p \leq 0.05 \vee s$ LC SEX; ${ }^{* *} p=0.06 v s 18 / 12$ S Sed

Figure 4: Effects of fish oil on the trabecular parameters of the distal femoral metaphysis of middle aged C57BI/6 mice ( $\mu$ CT): (i) trabecular thickness; (ii) trabecular number; (iii) Trabecular separation. (ap $\leq 0.05$ vs. LC S Sed; bp $\leq 0.05$ vs. $18 / 12$ S Sed; ${ }^{*} p \leq 0.05$ vs. LC S EX; ** $p=0.06$ vs. $18 / 12$ S Sed). 
Citation: Banu J, Fernandes G (2017) Fish Oil with Higher DHA Content and Voluntary Exercise Decreases Postmenopausal Bone Loss. J Osteopor Phys Act 5: 188. doi: 10.4172/2329-9509.1000188

Page 5 of 5

Whether the fatty acid is beneficial or detrimental to bone health is dependent on the type of fatty acid that is consumed [17]. Fatty acids may influence bone using different pathways including inflammatory and oxidative stress related pathways. Some fatty acids like n- 6 fatty acids enhance inflammation, and others such as n-3 fatty acids can reduce inflammation and counter oxidative stress $[18,19]$. However, the ratio of n-6/n-3 fatty acids has a major effect and can protect bone in animal models and humans [20-27]. The major fatty acids in FO, EPA and DHA are both beneficial to bone but the ratio of these n-3 fatty acids plays an important role in determining the extent of the benefits.

In addition to FO, EX also is implicated in helping increased bone mass by stimulating bone formation and reducing bone resorption [11]. In the present study, we tested two ratios of EPA/DHA along with exercise. We observed that FO 30/20 with higher concentrations of EPA and DHA could reduce bone loss mainly in the trabecular bone compartment of the distal femur. Exercise further reduced bone loss in the presence of 30/20. Cortical BMC was also increased in 30/20 FOfed mice. This is in line with several reports that support the beneficial effects of FO on bone [6-10,24], although, this is the first report that tested two concentrations of EPA and DHA.

In conclusion, FO-fed mice had increased bone mass in the distal femur; both fish oils showed higher trabecular number while 30/20 FO showed more decreased trabecular separation. It is probable that higher concentrations of EPA/DHA along with exercise may be more beneficial to bone. More extensive work must be conducted to understand the mechanism behind this influence of FO on bone.

\section{Conflict of Interest}

The authors declare that they have no conflict of interest.

\section{Acknowledgement}

This study was supported by grants from NIH (ROIAG023648-01A1, ROIAT004259-03). We also thank Ms. Nishu Kazi who helped us with feeding the mice and during the sacrifice. We acknowledge Ms. Erika Varela and Mr. Al Bahadur for the $\mu \mathrm{CT}$ densitometry.

\section{References}

1. Seeman E (2003) Periosteal bone formation--a neglected determinant of bone strength. N Engl J Med 349: 320-323.

2. Foidart JM, Desreux J, Pintiaux A, Gompel A (2007) Hormone therapy and breast cancer risk. Climacteric 10 Suppl 2: 54-61.

3. Valverde $P$ (2008) Pharmacotherapies to manage bone loss-associated diseases: A quest for the perfect benefit-to-risk ratio. Curr Med Chem 15: 284 304

4. Marx RE, Cillo JE Jr, Broumand V, Ulloa JJ (2008) Outcome analysis of mandibular condylar replacements in tumor and trauma reconstruction: A prospective analysis of 131 cases with long-term follow-up. J Oral Maxillofac Surg 66: 2515-2523.

5. Marx RE (2008) Bisphosphonate-induced osteonecrosis of the jaws: A challenge, a responsibility and an opportunity. Int J Periodontics Restorative Dent 28: 5-6.

6. Rahman MM, Bhattacharya A, Banu J, Kang JX, Fernandes G (2009) Endogenous $\mathrm{n}-3$ fatty acids protect ovariectomy induced bone loss by attenuating osteoclastogenesis. J Cell Mol Med 13: 1833-1844.

7. Rahman MM, Bhattacharya A, Fernandes G (2008) Docosahexaenoic acid is more potent inhibitor of osteoclast differentiation in RAW 264.7 cells than eicosapentaenoic acid. J Cell Physiol 214: 201-209.

8. Watkins BA, Li Y, Lippman HE, Feng S (2003) Modulatory effect of omega-3 polyunsaturated fatty acids on osteoblast function and bone metabolism. Prostaglandins Leukot Essent Fatty Acids 68: 387-398.
9. Watkins BA1, Li Y, Lippman HE, Seifert MF (2001) Omega-3 polyunsaturated fatty acids and skeletal health. Exp Biol Med 226: 485-497.

10. Poulsen RC, Wolber FM, Moughan PJ, Kruger MC (2008) Long chain polyunsaturated fatty acids alter membrane-bound RANK-L expression and osteoprotegerin secretion by MC3T3-E1 osteoblast-like cells. Prostaglandins Other Lipid Mediat 85: 42-48.

11. Banu MJ, Orhii PB, Mejia W, McCarter RJ, Mosekilde L, et al. (1999) Analysis of the effects of growth hormone, voluntary exercise and food restriction on diaphyseal bone in female F344 rats. Bone 25: 469-480.

12. Barengolts El, Curry DJ, Bapna MS, Kukreja SC (1993) Effects of endurance exercise on bone mass and mechanical properties in intact and ovariectomized rats. J Bone Miner Res 8: 937-942.

13. Yeh JK, Aloia JF, Chen M (1994) Growth hormone administration potentiates the effect of treadmill exercise on long bone formation but not on the vertebrae in middle-aged rats. Calcif Tissue Int 54: 38-43.

14. Yeh JK, Aloia JF, Chen M, Ling N, Koo HC, et al. (1994) Effect of growth hormone administration and treadmill exercise on serum and skeletal IGF-I in rats. Am J Physiol 266: E129-135.

15. Banu J, Wang L, Kalu DN (2003) Effects of increased muscle mass on bone in male mice overexpressing IGF-I in skeletal muscles. Calcif Tissue Int 73 196-201.

16. Corwin RL (2003) Effects of dietary fats on bone health in advanced age Prostaglandins Leukot Essent Fatty Acids 68: 379-386.

17. Raisz LG, Alander CB, Simmons HA (1989) Effects of prostaglandin E3 and eicosapentaenoic acid on rat bone in organ culture. Prostaglandins 37: 615625

18. Calder PC1 (2006) n-3 polyunsaturated fatty acids, inflammation and inflammatory diseases. Am J Clin Nutr 83: 1505S-1519S.

19. Wauquier F, Léotoing L, Philippe C, Spilmont M, Coxam V, et al. (2015) Pros and cons of fatty acids in bone biology. Prog Lipid Res 58: 121-145.

20. Banu J, Bhattacharya A, Rahman M, Kang JX, Fernandes G (2010) Endogenously produced $n-3$ fatty acids protect against ovariectomy induced bone loss in fat-1 transgenic mice. J Bone Miner Metab 28: 617-626.

21. Lau BY1, Ward WE, Kang JX, Ma DW (2009) Femur EPA and DHA are correlated with femur biomechanical strength in young fat-1 mice. J Nutr Biochem 20: 453-461.

22. Wauquier F, Barquissau V, Léotoing L, Davicco MJ, Lebecque $P$, et al. (2012) Borage and fish oils lifelong supplementation decreases inflammation and improves bone health in a murine model of senile osteoporosis. Bone 50: 553561.

23. Claassen N, Potgieter HC, Seppa M, Vermaak WJ, Coetzer H, et al. (1995) Supplemented gamma-linolenic acid and eicosapentaenoic acid influence bone status in young male rats: Effects on free urinary collagen crosslinks, total urinary hydroxyproline and bone calcium content. Bone 16: 385S-392S.

24. Watkins BA, Li Y, Seifert MF (2006) Dietary ratio of $n-6 / n-3$ PUFAs and docosahexaenoic acid: Actions on bone mineral and serum biomarkers in ovariectomized rats. J Nutr Biochem 17: 282-289.

25. Mollard RC, Kovacs HR, Fitzpatrick-Wong SC, Weiler HA (2005) Low levels of dietary arachidonic and docosahexaenoic acids improve bone mass in neonatal piglets, but higher levels provide no benefit. J Nutr 135: 505-512.

26. Weiler HA, Fitzpatrick-Wong SC (2002) Modulation of essential (n-6):(n-3) fatty acid ratios alters fatty acid status but not bone mass in piglets. J Nutr 132 2667-2672.

27. Högström M, Nordström P, Nordström A (2007) n-3 Fatty acids are positively associated with peak bone mineral density and bone accrual in healthy men the NO2 Study. Am J Clin Nutr 85: 803-807. 